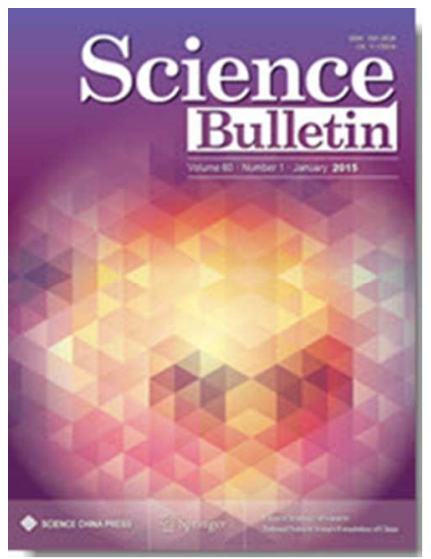

Discovery of a high-altitude ecotype and ancient lineage of Arabidopsis thaliana from Tibet

\begin{tabular}{|c|c|}
\hline Journal: & Science Bulletin \\
\hline Manuscript ID & CSB-2017-0708 \\
\hline Manuscript Type: & Short Communication \\
\hline $\begin{array}{r}\text { Date Submitted by the } \\
\text { Author: }\end{array}$ & 26-Jul-2017 \\
\hline Complete List of Authors: & $\begin{array}{l}\text { Zeng, Liyan; Fudan University, School of Life Science } \\
\text { Gu, Zhuoya; Fudan University } \\
\text { Xu, Min; Tibet University } \\
\text { Zhao, Ning; Tibet University } \\
\text { Yonezawa, Takahiro; Fudan University } \\
\text { Zhu, Weidong; Tibet University } \\
\text { Liu, Tianmeng; Tibet University } \\
\text { Zhang, Yang; university of Illinois at Urbana-Champaign } \\
\text { Qiong, Lha; Tibet University } \\
\text { Tersing, Tashi; Tibet University } \\
\text { Xu, Lingli; Fudan University, School of Life Science } \\
\text { Xu, Rongyan; Fudan University } \\
\text { Sun, Ningyu; Fudan University } \\
\text { Huang, Yanyan; Fudan University } \\
\text { Lei, Jiankun; Fudan University } \\
\text { Zhang, Liang; Fudan University } \\
\text { Xie, Feng; Soochow University } \\
\text { Zhang, Fang; 中科院遗传与发育生物学研究所 }\end{array}$ \\
\hline
\end{tabular}




\begin{tabular}{|r|l|}
\hline & Gu, Hongya; 北京大学生命科学学院 \\
& Geng, Yu peng; Yunnan University \\
& Hasegawa, Masami; Fudan University, School of Life Sciences \\
& Yang, Ziheng; University College London \\
& Crabbe, M. James C.; University of Oxford \\
& Chen, Fan; 中科院遗传与发育生物学研究所 \\
& Zhong, Yang; 复旦大学生命科学院, \\
\hline Keywords: & $\begin{array}{l}<\mathrm{i}>\text { Arabidopsis thaliana }</ \mathrm{i}>\text {, divergence time, Qinghai-Tibet } \\
\text { Plateau }\end{array}$ \\
\hline Speciality: & Life \& Medical Sciences/Plant Sciences \\
\hline & \\
\hline
\end{tabular}

SCHOLARONE ${ }^{m}$

Manuscripts 


\section{Discovery of a high-altitude ecotype and ancient lineage of Arabidopsis thaliana from Tibet}

Liyan Zeng ${ }^{1,2,3}+$, Zhuoya $\mathrm{Gu}^{2}+$, Min $\mathrm{Xu}^{1,4}+$, Ning Zhao ${ }^{1}+$, Weidong Zhu ${ }^{1}$, Takahiro Yonezawa ${ }^{2,5}$, Tianmeng Liu ${ }^{1}$, Lha Qiong ${ }^{1}$, Tashi Tersing ${ }^{1,6}$, Lingli $\mathrm{Xu}^{2}$, Yang Zhang ${ }^{7}$, Rongyan $\mathrm{Xu}^{2}$, Ningyu $\mathrm{Sun}^{2}$, Yanyan Huang ${ }^{2}$, Jiankun Lei ${ }^{8}$, Liang Zhang ${ }^{8}$, Feng Xie ${ }^{9}$, Fang Zhang ${ }^{10}$, Hongya $\mathrm{Gu}^{11}$, Yupeng Geng ${ }^{12}$, Masami Hasegawa ${ }^{2,5}$, Ziheng Yang ${ }^{13}$, M. James C. Crabbe ${ }^{14,15}$, Fan Chen ${ }^{10,}$, Yang Zhong ${ }^{1,2,16, *}$

1. Institute of Biodiversity Science and Geobiology, College of Sciences, Tibet University, Lhasa 850000, China

2. Ministry of Education Key Laboratory for Biodiversity Science and Ecological Engineering, School of Life Sciences, Fudan University, Shanghai 200433, China

3. Center for Clinical Public Health, Fudan University, Shanghai 201508, China

4. Institute of Forest Inventory, Planning and Research of Tibet Autonomous Region, Lhasa 850010 , China

5. Institute of Mathematical Statistics, Midori-cho 10-3, Tachikawa, Tokyo 190-8562, Japan

6. Tibet Museum of Natural Science, Lhasa 850000, China

7. Department of Bioengineering, University of Illinois at Urbana-Champaign, Champaign, IL, 61801, USA

8. School of Computer Science, Fudan University, Shanghai 200433, China

9. School of Urban Rail Transportation, Soochow University, Suzhou 215131, China

10. Institute of Genetics and Developmental Biology, Chinese Academy of Sciences, Beijing 100101, China

11. School of Life Sciences, Peking University, Beijing 100871, China

12. School of Life Sciences, Yunnan University, Kunming 650091, China

13. Department of Genetics, Evolution and Environment, University College London, Darwin Building, Gower Street, London WC1E 6BT, United Kingdom

14. Department of Zoology, University of Oxford, Tinbergen Building, South Parks Road, Oxford, OX1 3PS, United Kingdom 
15. Institute of Biomedical and Environmental Science \& Technology, Department of Life Sciences, University of Bedfordshire, Park Square, Luton, LU1 3JU, United

\section{Kingdom}

16. Shanghai Center for Bioinformation Technology, Shanghai 201203, China

†These authors contributed equally to this work

*Correspondence to: Fan Chen \& Yang Zhong 
Arabidopsis thaliana has long been a model species for dicotyledon study, and was the first flowering plant to get its genome completed sequenced [1]. Although most wild $A$. thaliana are collected in Europe, several studies have found a rapid $A$. thaliana west-east expansion from Central Asia [2]. The Qinghai-Tibet Plateau (QTP) is close to Central Asia and known for its high altitude, unique environments and biodiversity[3]. However, no wild-type A. thaliana had been either discovered or sequenced from QTP. Studies on the A. thaliana populations collected under $2000 \mathrm{~m}$ asl have shown that the adaptive variations associated with climate and altitudinal gradients [4]. Hence a high-altitude $A$. thaliana provides a precious natural material to investigate the evolution and adaptation process.

Here, we present the genome of a new ecotype of $A$. thaliana collected in the Gongga County, Tibet (4200 m asl) (Fig. 1A), to demonstrate its evolutionary history and adaptation to high-altitude regions. The Tibetan samples were identified as $A$. thaliana by comparing the nuclear internal transcribed spacer (ITS), four chloroplast genes $(m a t \mathrm{~K}, r b c \mathrm{~L}, r p o \mathrm{~B}$, and $r p s 16)$, and three chloroplast intergenic spacers (IGS,

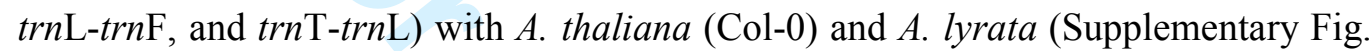
1). This is the first report that an A. thaliana population has been collected in the QTP over $4000 \mathrm{~m}$ asl and identified by molecular analysis. Moreover, the new Tibetan ecotype (herein referred to as "Tibet- 0 ") is diploid $(2 n=10)$ according to karyotype analysis of its pollen mother cells during meiosis (Supplementary Fig. 2), suggesting that the ploidy of the Tibet- 0 is stable and capable of further sequence analysis.

We then conducted genome-wide resequencing of Tibet- 0 with a mean coverage of 40x of the reference genomes Col-0 and TAIR10, by using Illumina Hiseq2000 (Supplementary Table 5-6). We compared Tibet-0 with 47 other $A$. thaliana ecotypes that have been genome-wide sequenced, and found that Tibet-0 was of high divergence, including a higher proportion of SNPs (Supplementary Table 7-9). Evolutionary relationships between Tibet- 0 and other ecotypes were evaluated by the following two independent approaches based on 5611 single-copy orthologues in $47 \mathrm{~A}$. thaliana ecotypes including 26 relicts and 21 non-relicts defined by the 1001 Genomes Consortium[5]. The first approach is the phylogenetic method. The genealogy among the individuals were inferred based on the concatenated genomic data, and Tibet- 0 was placed at the root of the $A$. thaliana populations with high support value (Fig. 1B) [5, 6]. It makes Tibet- 0 the most ancestral lineage.

However, since this phylogenetic approach assumes that all gene loci have the same genealogy, coalescent method was also applied as a cross check [7]. In this method, 2788 single-copy orthologues were independently analyzed, and the distributions of the tMRCAs (the time to the most recent common ancestor) for these genes were estimated. If Tibet- 0 is the most basal lineage among the A. thaliana populations and Tibet- 0 specific alleles has generally older histories than others, tMRCAs excluding Tibet- 0 will be smaller than tMRCAs of all A. thaliana populations. Otherwise, if there is no such genetic structure and Tibet-0 specific alleles are included within the genetic diversity of other $A$. thaliana populations, 
tMRCAs excluding Tibet-0 will be equal to the tMRCAs of all $A$. thaliana populations. To examine the differences among the distributions, the tMRCAs were first estimated based on $48 \mathrm{~A}$. thaliana (tMRCA48). Subsequently, each ecotype was excluded once, and the tMRCAs of 47 remaining $A$. thaliana were estimated (tMRCA47: there are 48 combinations of tMRCA47). Finally, the relative tMRCAs (tMRCA47/tMRCA48) were estimated. Fig. 1C illustrates the distributions of the relative tMRCAs. When Tibet- 0 was excluded, the distribution of the relative tMRCAs (tMRCA47 excluding Tibet-o/tMRCA48) significantly shifted ( $\mathrm{t}$ test, $\mathrm{p}=9.77 \mathrm{E}$ 32), while the average of tMRCA $47_{\text {excluding one ecotype other than Tibet- } 0 / \text { tMRCA48 showed no }}$ significant change (Fig. 1C). These findings confirm that Tibet- 0 has the most ancestral positions among $A$. thaliana populations.

To understand the correlation between the evolution of $A$. thaliana and major geological events, especially Tibetan uplifts, the divergence time between Tibet- 0 and other ecotypes were estimated. Since there is no suitable fossil calibrations within $A$. thaliana, the divergence time between A. lyrata and A. thaliana was estimated based on the genomic data in the framework of whole land plant evolution with reliable fossil records, and it was estimated to be about 9 million years ago (Fig. 1D, Supplementary Fig. 3). Then, the time of the common ancestor of A. thaliana was estimated by multiplying the divergence time between $A$. lyrata and $A$. thaliana and the ratio of the divergence time between $A$. lyrata and $A$. thaliana. The divergence time between Tibet- 0 and other ecotypes was found to be 126 - $149 \mathrm{Ka}$ (kili annum: thousand years ago). Interestingly, the Gonghe movement, which was the last phase of Tibetan uplift, isolated the Qinghai Lake and raised the QTP to its present height algo began at about $15 \mathrm{Ka}$ [8]. Besides, the divergence time of Tibet- 0 and other ecotypes is in the middle Pleistocene from 781 to $126 \mathrm{Ka}$ [9].

A. thaliana has been widely used in studies of plant biology. By collecting and sequencing $A$. thaliana collected from the QTP over $4200 \mathrm{~m}$ asl, we have found that the Tibet- 0 is a new and divergent ecotype that isolated from other A. thaliana ecotypes since the last uplift of the QTP. After 126 - 149 thousands years evolution in the extreme plateau environment, Tibet- 0 possesses a distinctive genome with a high proportion of SNPs compared to other ecotypes. According to the strongly negatively skewed Tajima's D of 5611 single-copy orthologues, a recent selective sweep or population expansion might have occurred in the A. thaliana, which is consistent with previous studies (Supplementary Fig. 6)[10]. Considering the ancestral position of Tibetan populations as well as the subsequent selective sweep or population expansion, possibly in the Last Glacial Period, suggested by the negative Tajima's D, we suppose that some mutations might have emerged in the ancient A. thaliana population located around the QTP, and then spread to most other populations. Following step is investigating phenotypic traits of Tibet- 0 to study the adaptive evolution of $A$. thaliana to high altitudes. As a new model plant, the Tibet-0 from QTP would provide an invaluable material for further study. 


\section{Declarations}

\section{Acknowledgements}

This study was supported by the National Natural Science Foundation of China (91131901), the specimen platform of China (teaching specimens sub-platform) and PSCIRT project.

\section{Availability of data and materials}

The genomic DNA of Tibet-0 have been deposited in the Sequence Read Archive (SRA, http://www.ncbi.nlm.nih.gov/sra/) under accession number SRP052218.

\section{Author Contributions}

F. C. and Y. Z. conceived the project. L. Zeng, Z. G., T. Y., F. C. and Y. Z. contributed to the design of the project and extensive discussions. M. X., N. Z., W. Z., L. Q. and T. T. collected samples from Tibet. L. Zeng and H. G. helped with sample identification. L. X., R., X., F. X., J. L., L. Z., Z. G., N. Z., Y. H., T. Y., M. H., F. Z., F. C., Y. G., L. Zhang, Z. Y., M. J. C. C. and Y. Z. performed the common garden experiments, sequence analyses and evolutionary analyses. L. Zeng, Z. G., M. J. C. C., N. S., F. C. and Y. Z. wrote the manuscript. Other authors revised the manuscript.

Correspondence and requests for the materials should be addressed to F.C. (fchen@genetics.ac.cn) or Y. Z. (yangzhong@,fudan.edu.cn).

\section{Competing interests}

The authors declare no competing financial interests.

\section{Ethics approval and consent to participate}

Not applicable

\section{Supplementary Materials:}

Figures S1-S4

Table S1-S9 


\section{Reference:}

1. Arabidopsis Genome, I., Analysis of the genome sequence of the flowering plant Arabidopsis thaliana. Nature, 2000. 408(6814): p. 796-815.

2. Yin, P., et al., The origin of populations of Arabidopsis thaliana in China, based on the chloroplast DNA sequences. BMC Plant Biol, 2010. 10: p. 22.

3. Liu S, W.N., Duan K, Xiao C, Ding Y, Recent progress of glaciological studies in China. Journal of Geographical Sciences, 2004. 14(4): p. 401-410.

4. Suter, L., et al., Gene regulatory variation mediates flowering responses to vernalization along an altitudinal gradient in Arabidopsis. Plant Physiol, 2014. 166(4): p. 1928-42.

5. Consortium, G., 1,135 Genomes Reveal the Global Pattern of Polymorphism in Arabidopsis thaliana. Cell, 2016. 166(2): p. 481-91.

6. Gan, X., et al., Multiple reference genomes and transcriptomes for Arabidopsis thaliana. Nature, 2011. 477(7365): p. 419-23.

7. Nakagome, S., S. Mano, and M. Hasegawa, Comment on "Nuclear genomic sequences reveal that polar bears are an old and distinct bear lineage". Science, 2013. 339(6127): p. 1522.

8. Li, J., Late Cenozoic intensive uplift of Qinghai-Xizang Plateau and its impacts on environments in surrounding area. Quaternary Science, 2001. 21: p. 381-391.

9. Cohen KM, G.P., Global chronostratigraphical correlation table for the last 2.7 million years. Subcommission on Quaternary Stratigraphy, 2011. 31(2): p. 243-247.

10. Shimizu, K.K., et al., Darwinian selection on a selfing locus. Science, 2004. 306(5704): p. 20814. 
A

C

- Tibet-0

- Other ecotypes

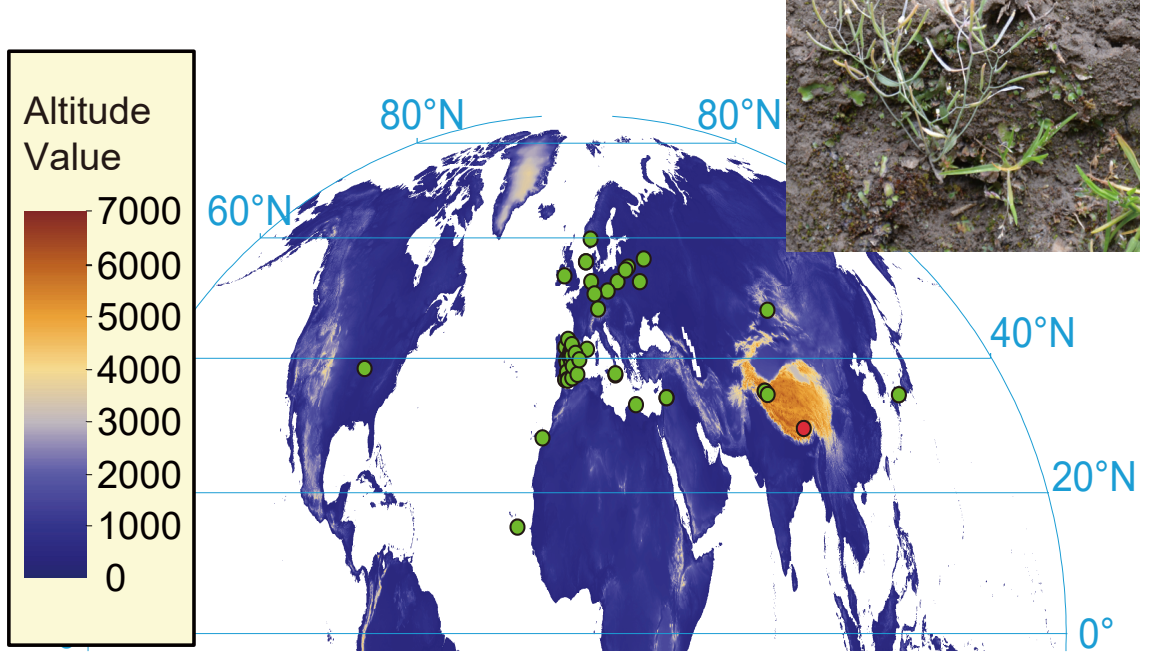

B

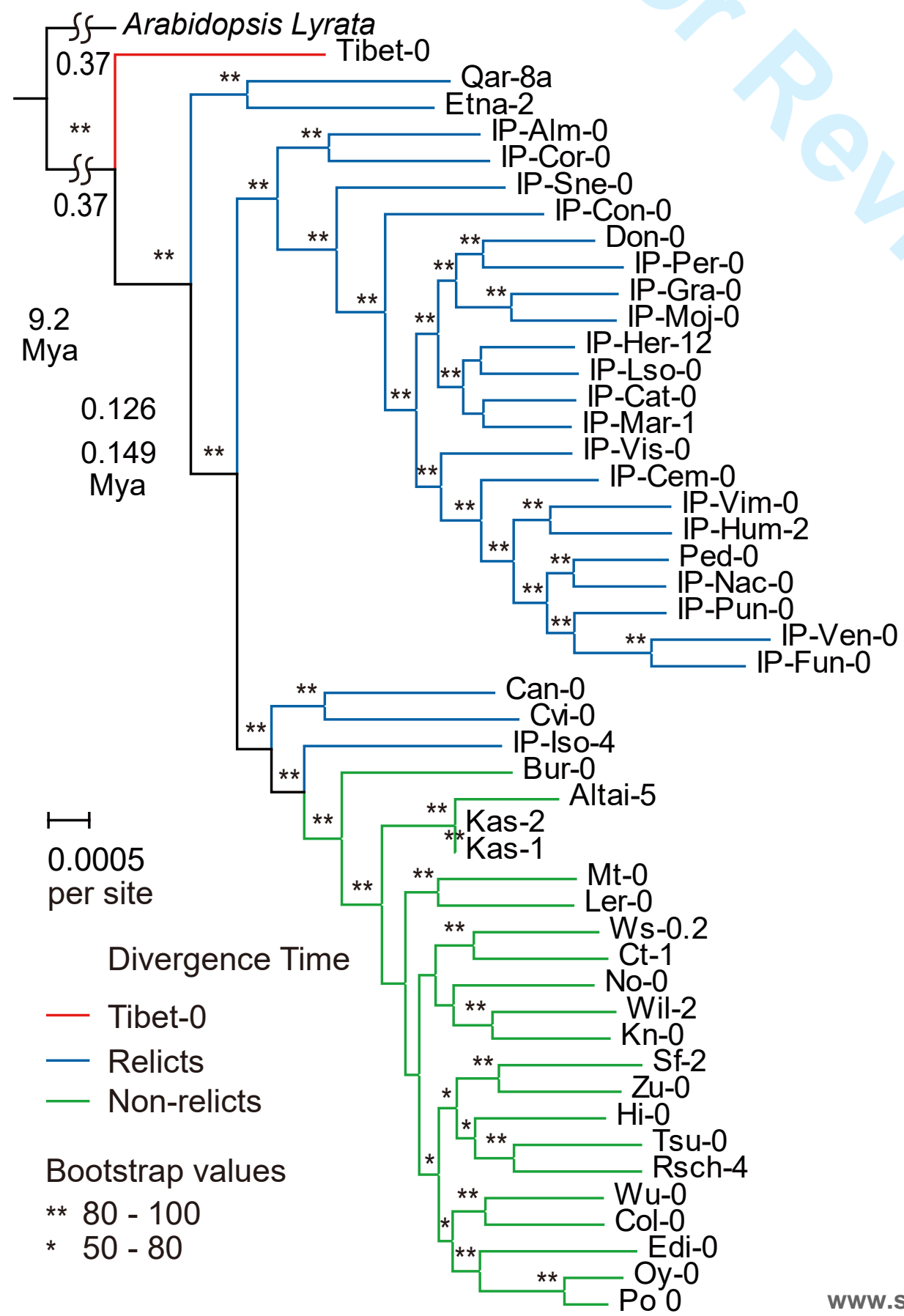

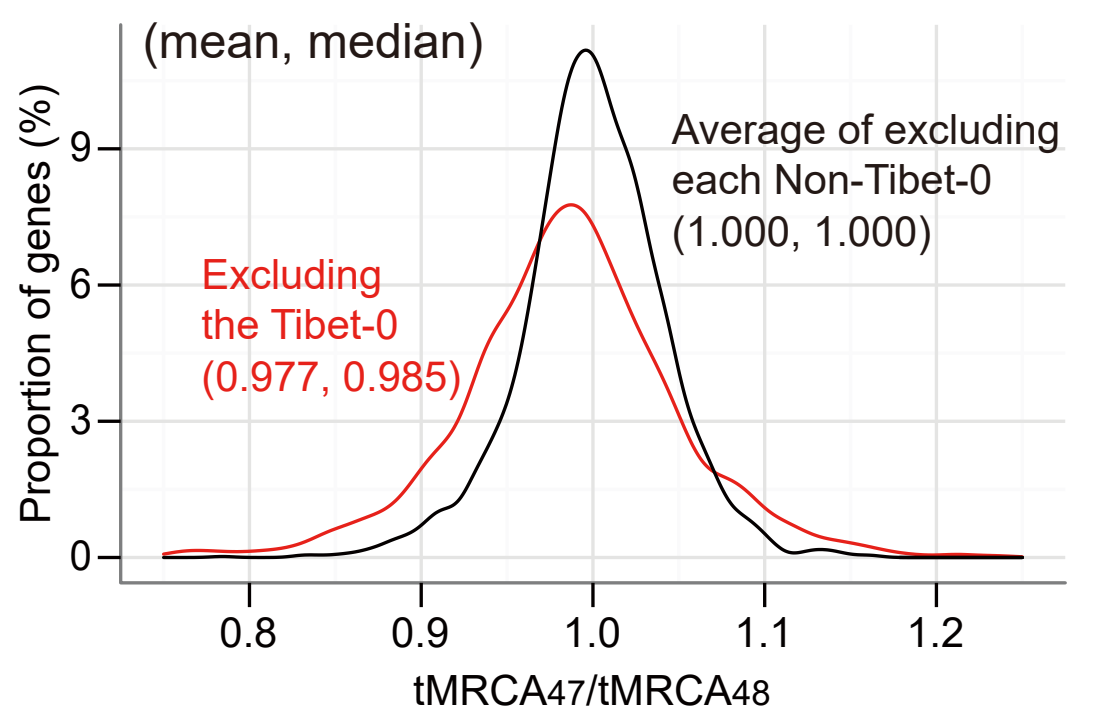

D

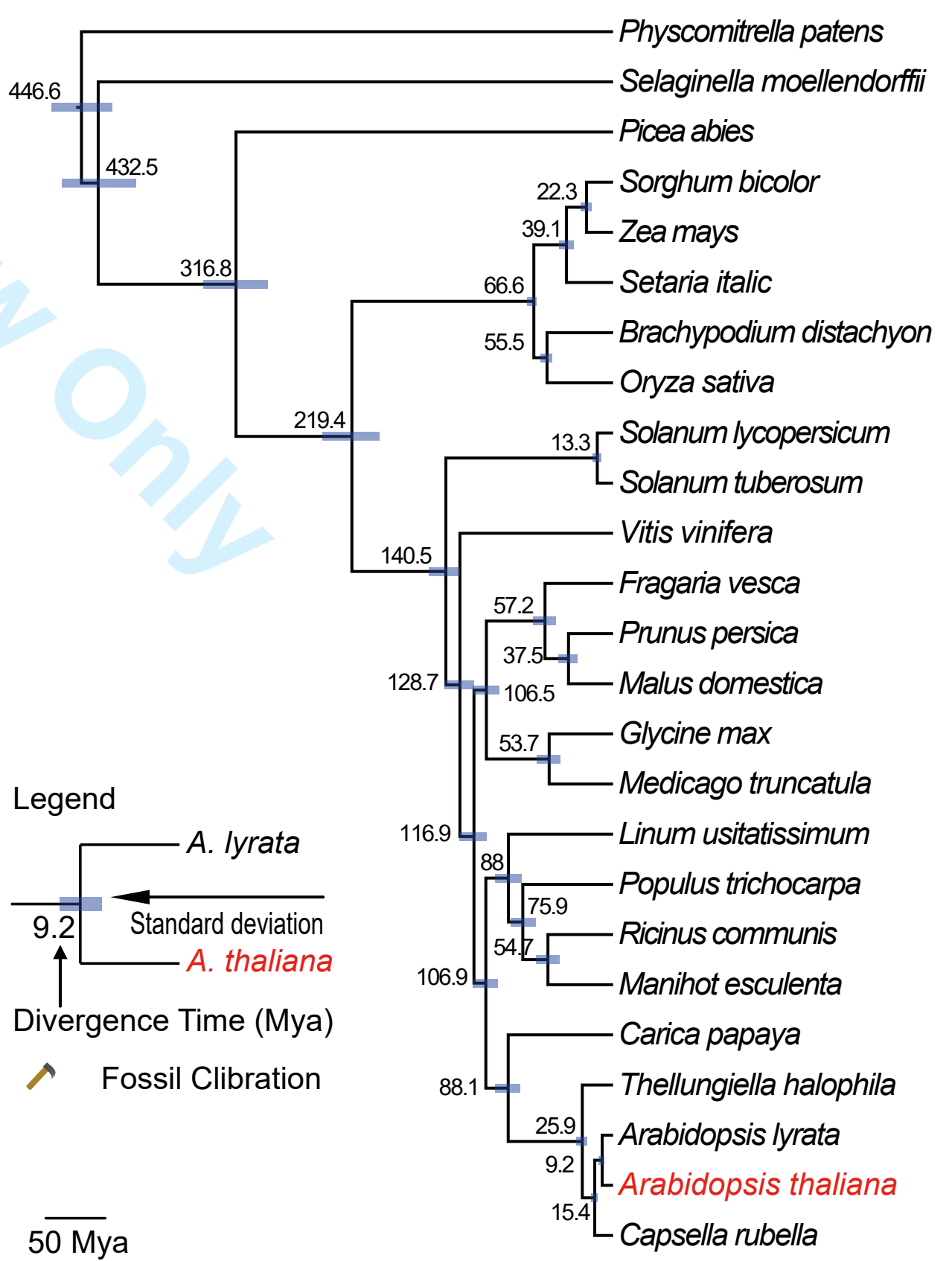


Figure 1. Collection, phylogentic analysis and adaptation analysis of the Tibet-0 and other 47 A. thaliana ecotypes.

A, Origins of Tibet- 0 and other $47 \mathrm{~A}$. thaliana ecotypes that we have analyzed in this paper (Supplementary Table 4). Elevation data were downloaded from WorldClim (http://www.worldclim.org/). Colors indicate altitudes, going from low to high elevation: Deep blue, blue, white, yellow, orange, and brown.

$B$, The maximum likelihood phylogenetic trees based on 5611 orthologues of Tibet- 0 , 47 A. thaliana ecotypes and $A$. lyrata used as outgroup. Bootstrap values based on 100 replications are listed as percentages at each node. The Tibet- 0 was marked in red.

C, The time of most recent common ancestor (tMRCA) based on 2788 single-copy orthologues. The red line represents the tMRCA47/tMRCA48 distribution where the tMRCA47 values exclude Tibet- 0 . The black line represents the mean of tMRCA47/tMRCA48 distribution where the tMRCA47 values exclude each Non-Tibet0 .

$D$, Phylogenetic affinities inferred from the maximum likelihood analysis of nucleotide sequence of 334 single copy orthologues in 25 plants. The divergence time of A. thaliana and A. lyrata was about 9.2Mya (million years ago). 7 fossil calibrations used in the study were marked as the hammer symbol. Branch lengths are proportional to the number of expected nucleotide substitutions. The number on the branch is the divergence time and unit is Mya. 
A

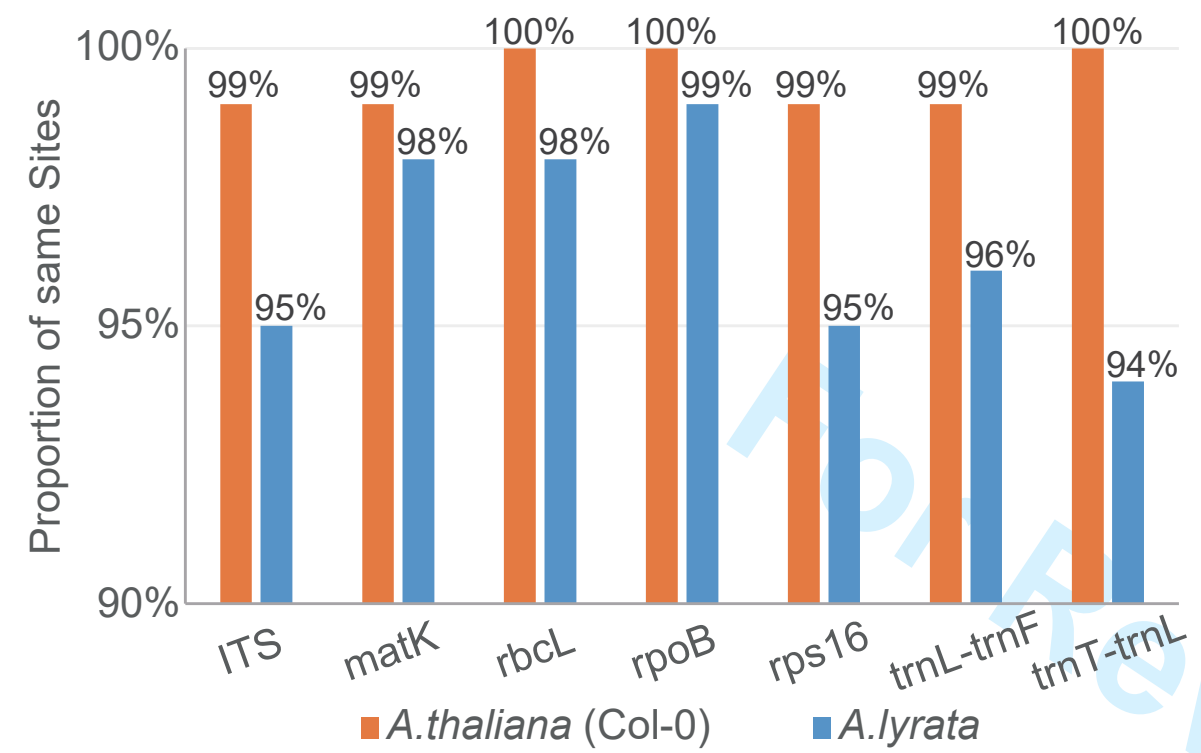


Figure S1. Gene barcoding comparison of Tibet-0 with A. thaliana and A. Iyrata.

A. The barcoding genes were nuclear internal transcribed spacer (ITS), four chloroplast genes (matK, rbcL, rpoB, rps16) and two chloroplast intergenic spacers (IGS, trnL-trnF, trnT-trnL). 
A

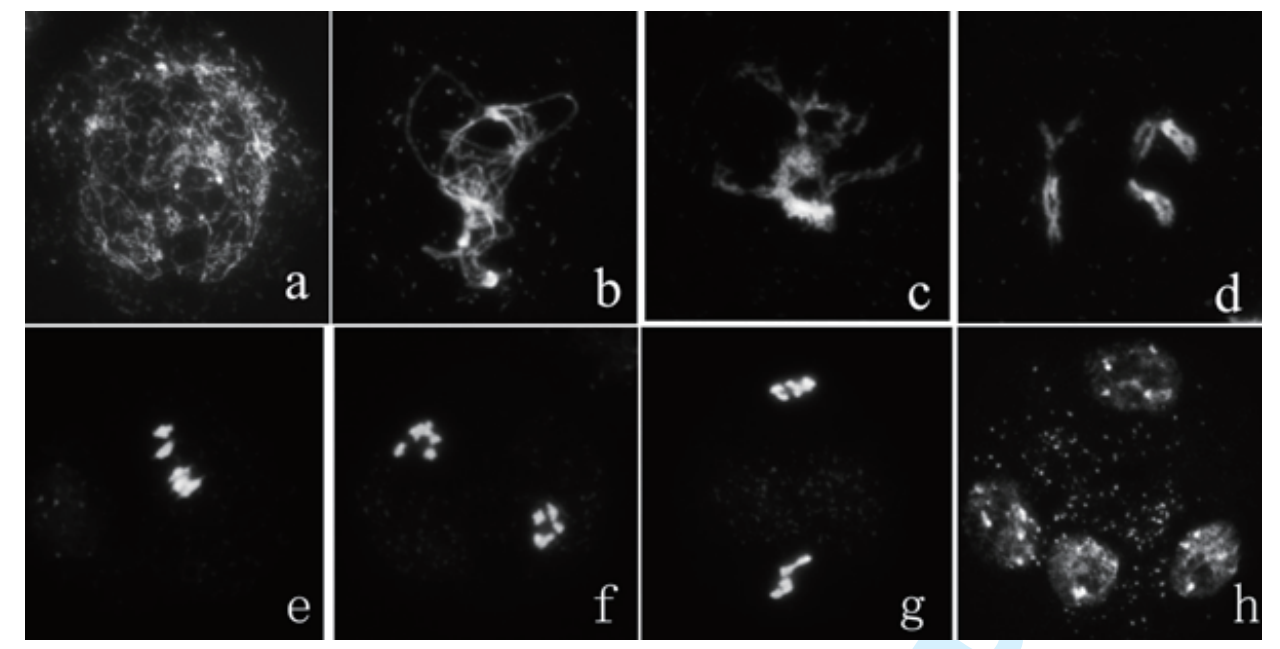

Tibet- $0 \mathrm{n}=2$
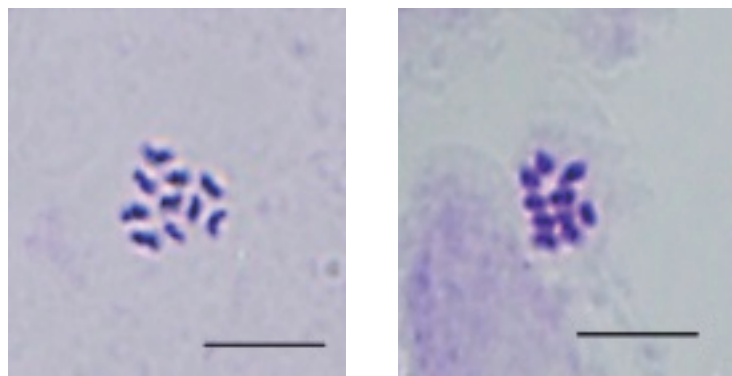

Col $n=2$

Tibet $-0 \mathrm{n}=2$

Bar $=1 \mathrm{um}$ 
1

2

3

Figure S2. Results of the polyploidy analysis of the Tibet-0.

A. Each phase of the meiosis of Tibet- 0 pollens was listed below. For each phase of Tibet0, a: leptotene; b: pachytene; c: diplotene; d: diakinesis; e: Metaphase I; f: Anaphase I; g: Metaphase II; h: tetrads stage.

B. Karyotypes of $A$. thaliana ecotype Col- 0 and Tibet- 0 . 


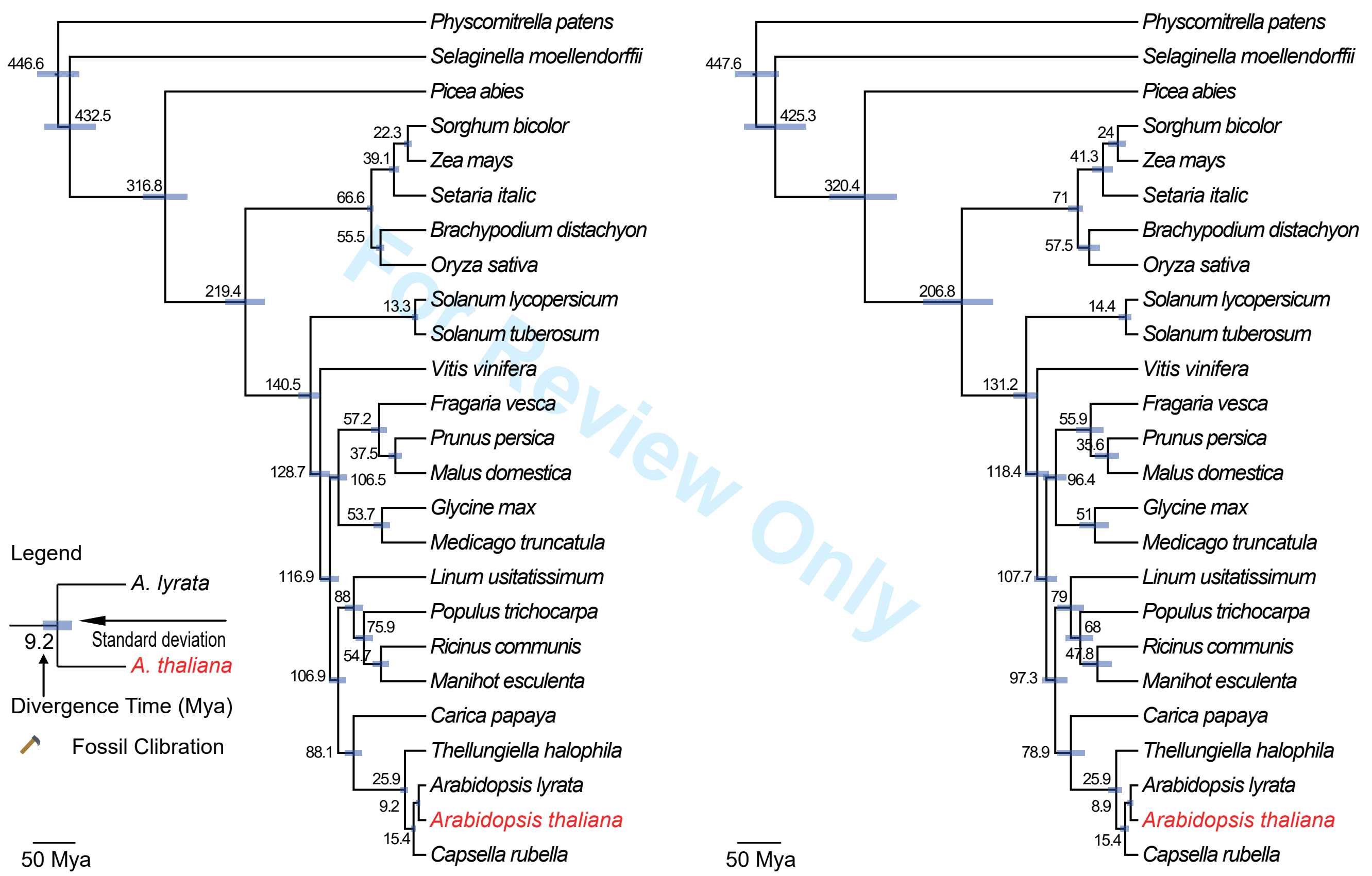


Figure S3. Results of divergence time estimation between A. thaliana and A. lyrata. A. Phylogenetic affinities inferred from the maximum likelihood analysis of nucleotide sequence of 334 single copy orthologues in 25 plants. The divergence time of $A$. thaliana and $A$. lyrata was about 9.2Mya (million years ago). 7 fossil calibrations used in the study were marked as the hammer symbol. Branch lengths are proportional to the number of expected nucleotide substitutions. The number on the branch is the divergence time and unit is Mya.

B. Phylogenetic affinities inferred from the maximum likelihood analysis of amino acid sequence of 334 single copy orthologues in 25 plants. The divergence time of $A$. thaliana and $A$. lyrata was about 8.9 Mya (million years ago). 7 fossil calibrations used in the study were marked as the hammer symbol. Branch lengths are proportional to the number of expected nucleotide substitutions. The number on the branch is the divergence time and unit is Mya. 
A

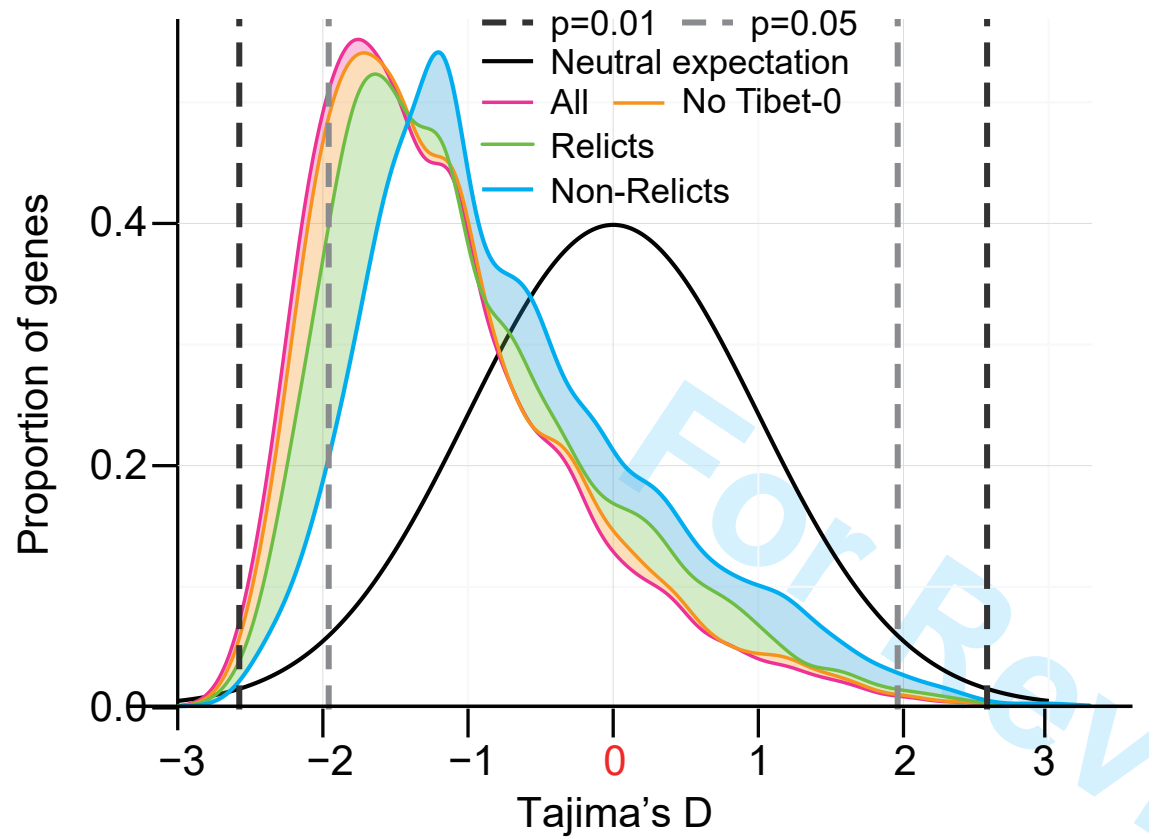


1

2

3

4

5

6

7

8

9

10

11

12

13

Figure S4. Results of the nucleotide diversity analysis of 48 A. thaliana ecotypes.

A. Distributions of Tajima's D statistic for each of the 5611 genes of $A$. thaliana populations. The black line shows the expected distribution of $D$ with no selection in a panmictic population of constant size. The red line shows the distribution of $D$ of 5611 genes of Tibet0 and other $47 A$. thaliana accessions. The yellow line shows the distribution of $D$ of 5611 genes of $47 A$. thaliana accessions that excluding Tibet- 0 . The green line shows the distribution of $D$ of 5611 genes of relicts. The blue line shows the distribution of $D$ of 5611 genes of non-relicts that exclude relicts and Tibet- 0 . 
Table S1. Amplification information of chloroplast and nuclear genes in Tibet-0 for barcoding

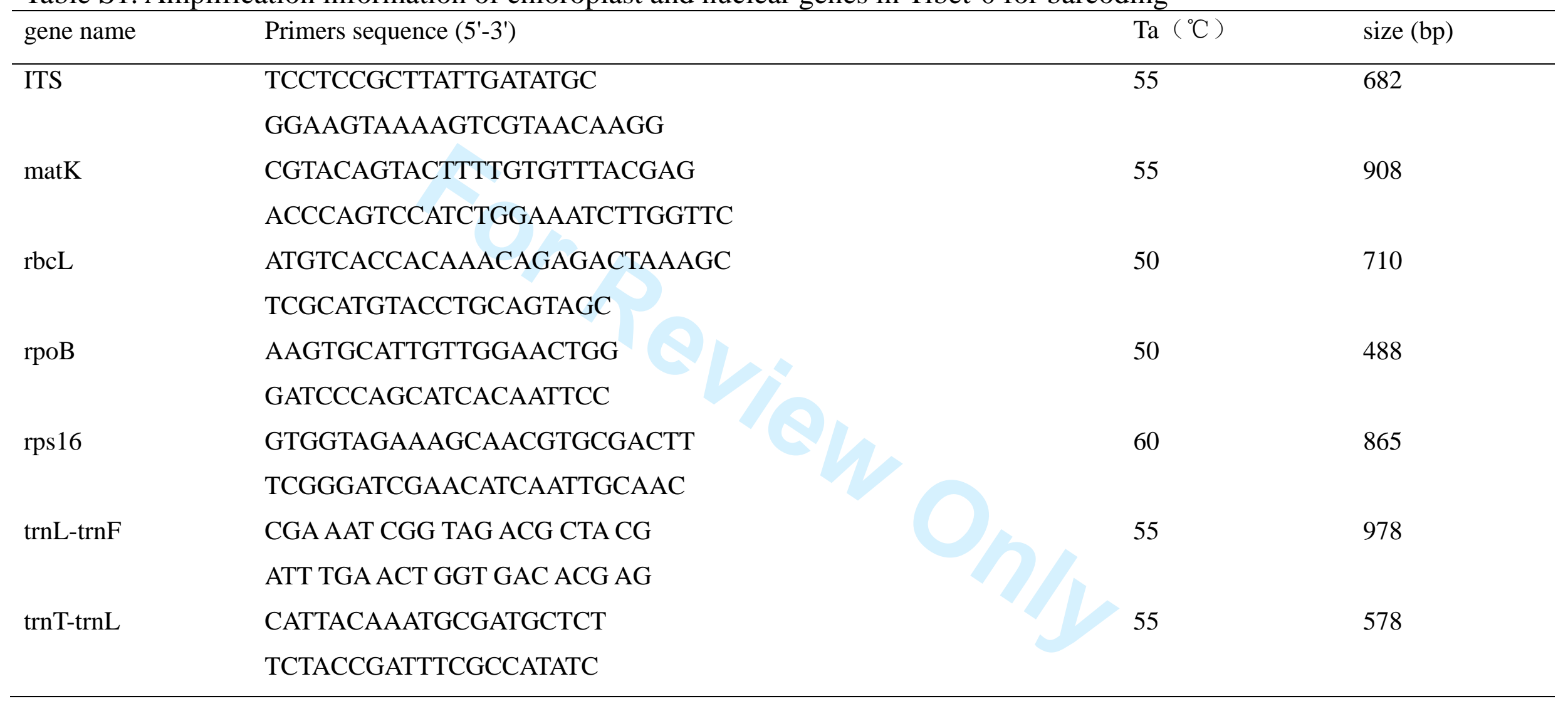


Table S2. Twenty five species used in divergence time estimation

\begin{tabular}{lll}
\hline Species & Order_APGIII & Sources \\
\hline Cucumissativus & Cucurbitales & Phytozome \\
Glycine max & Fabales & Phytozome \\
Medicagotruncatula & Fabales & Phytozome \\
Fragariavesca & Rosales & Phytozome \\
Malus domestica & Rosales & Phytozome \\
Pruspersicaun & Rosales & Phytozome \\
Manihotesculenta & Malpighiales & Phytozome \\
Ricinuscommunis & Malpighiales & Phytozome \\
Populustrichocarpa & Malpighialesi & Phytozome \\
Linumusitatissimum & Malpighiales & Phytozome \\
Arabidopsis thaliana & Brassicales & Phytozome \\
Arabidopsis lyrata & Brassicales & Phytozome \\
Capsella rubella & Brassicales & Phytozome \\
Carica papaya & Brassicales & Phytozome \\
Thellungiellahalophila & Brassicales & Phytozome \\
Vitisvinifera & Vitales & Phytozome \\
Solanum lycopersicum & Solanales & Phytozome \\
Solanum tuberosum & Solanales & Phytozome \\
Oryza sativa & Poales & Phytozome \\
Sorghum bicolor & Poales & Phytozome \\
Setariaitalica & Poales & Phytozome \\
Zea mays & Poales & Phytozome \\
Piceaabies & Gymnosperm & Spruce Genome Project \\
Selaginellamoellendorffii & Fern & Phytozome \\
Physcomitrella patens & Moss & Phytozome \\
\hline & & \\
\hline & & \\
\hline
\end{tabular}


Table S3. Seven fossil calibration used in divergence time estimation

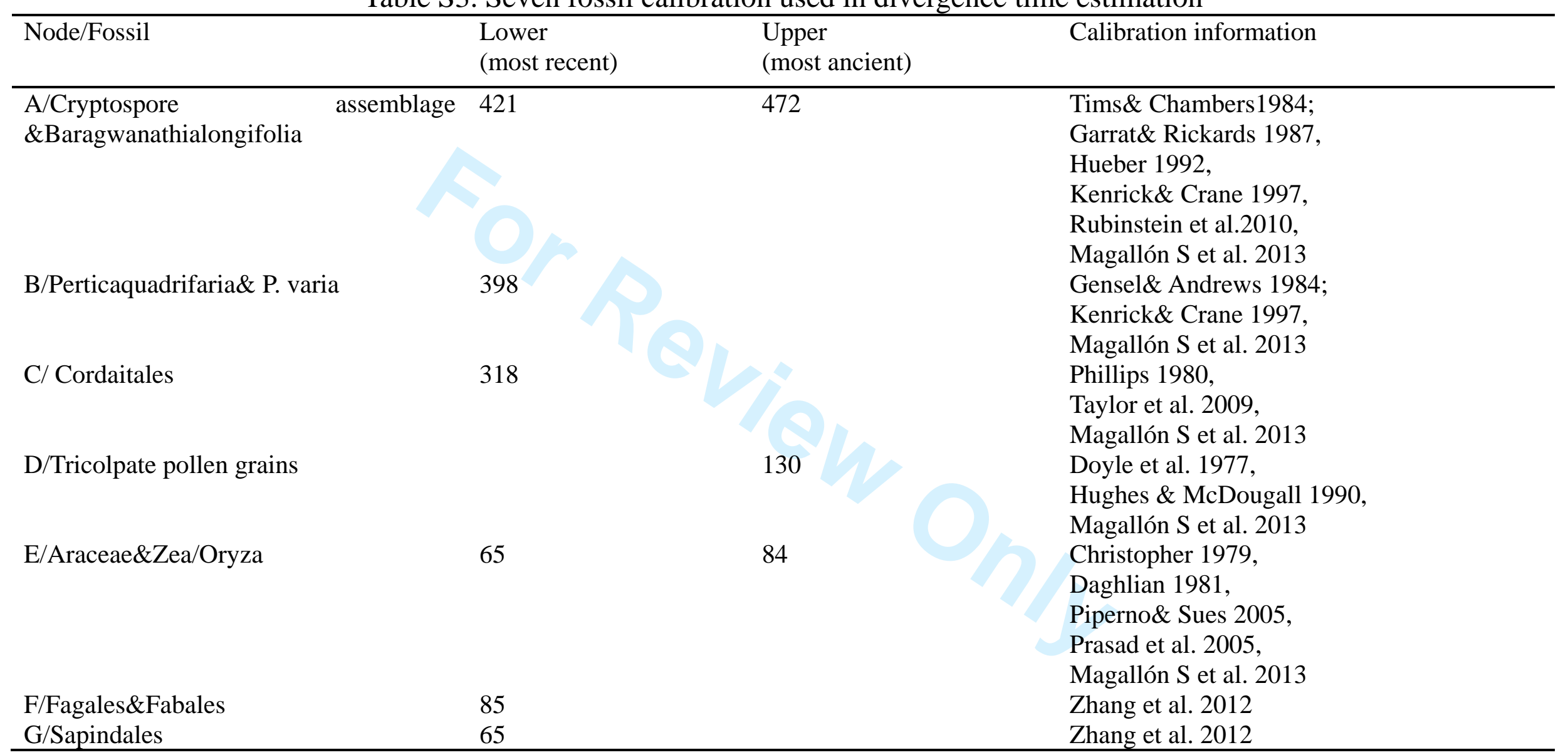


Table S4. The location and altitude of Tibet- 0 and 47 other ecotypes

\begin{tabular}{l|l|l|l}
\hline Sample & country & $\begin{array}{l}\text { Latitude } \\
(\mathrm{min} / \mathrm{max})\end{array}$ & $\begin{array}{l}\text { Longitude } \\
(\mathrm{min} / \mathrm{max})\end{array}$ \\
\hline Tibet-0 & China & N90.98 & E29.3 \\
\hline Ler-0 & Poland & N50 / N55 & E14.5 / E23 \\
\hline No-0 & Germany & N51 / N51 & E13 / E14 \\
\hline Wil-2 & Russia & N55 / N55 & E25 / E25 \\
\hline Kn-0 & Lithuania & N54 / N55 & E23 / E24 \\
\hline Ws-0 & Russia & N52 / N53 & E30 / E30 \\
\hline Ct-1 & Italy & N37 / N38 & E15 / E15 \\
\hline Rsch-4 & Russia & N56 / N57 & E34 / E34 \\
\hline Tsu-0 & Japan & N34 / N35 & E136 / E129 \\
\hline Hi-0 & Netherlands & N52 / N53 & E5 / E6 \\
\hline Oy-0 & Norway & N60 & E6 \\
\hline Po-0 & Germany & N50 / N51 & E7 / E7 \\
\hline Edi-0 & United Kingdom & N56 / N56 & E3 / E3 \\
\hline Wu-0 & Germany & / & / \\
\hline Col-0 & USA & Switzerland & W92 / W93 / N39 \\
\hline Zu-0 & & E8 / E9 \\
\hline
\end{tabular}




\begin{tabular}{|c|c|c|c|}
\hline Sf-2 & Spain & N41 / N42 & E3 / E3 \\
\hline Mt-0 & Libya & N33 / N33 & $\mathrm{E} 23$ / E23 \\
\hline Bur-0 & Ireland & N52 / N55 & W6 / W10 \\
\hline Can-0 & Spain & N28 / N28 & W15 / W15 \\
\hline Cvi-0 & Cabo Verde & N15 & W24 \\
\hline IP-Vim-0 & Spain & N41 & W7 \\
\hline Don-0 & Spain & N36 & W7 \\
\hline IP-Vis-0 & 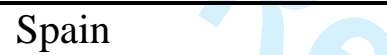 & N39 & W7 \\
\hline IP-Her-12 & Spain & N39 & W6 \\
\hline IP-Con-0 & Spain & N37 & W6 \\
\hline IP-Gra-0 & Spain & N36 & W6 \\
\hline IP-Iso-4 & Spain & N43 & W6 \\
\hline IP-Moj-0 & Spain & N36 & W6 \\
\hline IP-Pun-0 & Spain & N40 & W5 \\
\hline IP-Cem-0 & Spain & N41 & W5 \\
\hline IP-Fun-0 & Spain & $\mathrm{N} 40$ & W5 \\
\hline IP-Ven-0 & Spain & N40 & W5 \\
\hline IP-Nac-0 & Spain & N40 & W4 \\
\hline IP-Mar-1 & Spain & N39 & W4 \\
\hline
\end{tabular}




\begin{tabular}{l|l|l|l}
\hline Ped-0 & Spain & N40 & W4 \\
\hline IP-Cat-0 & Spain & N40 & W4 \\
\hline IP-Hum-2 & Spain & N42 & W4 \\
\hline IP-Sne-0 & Spain & N37 & W4 \\
\hline IP-Lso-0 & Spain & N38 & W4 \\
\hline IP-Cor-0 & Spain & N40 & W2 \\
\hline IP-Per-0 & Spain & N37 & W2 \\
\hline IP-Alm-0 & Spain & N39 & W1 \\
\hline Etna-2 & Italy & N37 & E14 \\
\hline Qar-8a & Lebanon & N34 & E35 \\
\hline Kas-1 & India & N35 & E77 \\
\hline Kas-2 & India & N35 & E77 \\
\hline Altai-5 & China & N47 & E88 \\
\hline
\end{tabular}


Table S5. Reads of the genome-wide resequencing of Tibet-0

\begin{tabular}{llllll}
\hline All Reads & Mapped Reads & Mapped Ratio(\%) & Unique mapped & Multiple mapped & Unmapped \\
\hline 109382230 & 100807134 & 0.92 & 77242012 & 23565122 & 8575096 \\
\hline & & & & \\
\hline
\end{tabular}


Table S6. Genome-wide comparison between Tibet-0 and Col-0

\begin{tabular}{|c|c|c|}
\hline Type & Reference (TAIR 10) & Tibet-0 \\
\hline genes & 28775 & 28647 \\
\hline gene models & 41671 & 41458 \\
\hline mRNA & 35386 & 35265 \\
\hline $\mathrm{CDS}$ & 35386 & 35237 \\
\hline pseudogenes & 924 & 906 \\
\hline pseudogenic_transcript & 926 & 908 \\
\hline transposable_element_gene & 3903 & 3855 \\
\hline mRNA_TE_GENE & 3911 & 3863 \\
\hline ncRNA & 480 & 474 \\
\hline miRNA & 180 & 179 \\
\hline rRNA & 15 & 15 \\
\hline snoRNA & 71 & 71 \\
\hline snRNA & 13 & 13 \\
\hline tRNA & 689 & 683 \\
\hline total & 152330 & 151574 \\
\hline
\end{tabular}


Table S7. Genome-wide SNPs of Tibet-0

\begin{tabular}{|c|c|c|c|c|c|c|c|c|c|c|}
\hline & SNP & Intergenic & Upstream & 5' UTR & CDS & Intron & 3' UTR & Downstream & Synonymous & Non-synonymous \\
\hline Chr2 & 218482 & 107499 & 23839 & 3215 & 30148 & 24706 & 5379 & 16241 & 15840 & 14030 \\
\hline Chr4 & 218243 & 97723 & 22232 & 3143 & 37284 & 28251 & 5817 & 15398 & 19782 & 17131 \\
\hline Chr5 & 279959 & 105586 & 32712 & 4575 & 52784 & 39795 & 8211 & 23600 & 27844 & 24514 \\
\hline ChrM & 74 & 23 & 14 & 2 & 19 & 6 & 0 & 4 & 5 & 14 \\
\hline ChrC & 59 & 1 & 12 & 0 & 21 & 4 & 0 & 5 & 11 & 10 \\
\hline
\end{tabular}

Table S8. Genome-wide Indels of Tibet- 0

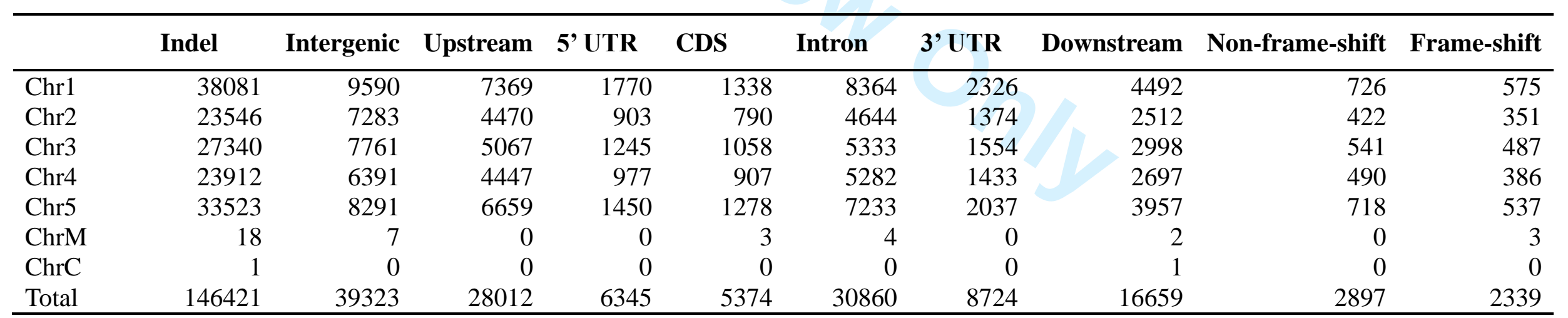


Table S9. SNPs and RNA-seq information of 19 A. thaliana sequences. (Gan et al. 2011)[1]

\begin{tabular}{|c|c|c|c|c|c|}
\hline \multirow[b]{2}{*}{ Accession } & \multicolumn{2}{|c|}{ Nucleotide differences } & \multicolumn{3}{|c|}{ RNA-seq support (SNPs) } \\
\hline & SNPs & $\begin{array}{l}\text { Ambiguous } \\
\text { positions }\end{array}$ & Agree & Disagree & \% concordance \\
\hline Can-0 & 789,187 & 54,148 & 118,965 & 343 & 99.7125 \\
\hline Bur-0 & 673,965 & 51,113 & 101,981 & 248 & 99.7574 \\
\hline Ct-1 & 650,332 & 44,342 & 100,175 & 271 & 99.73 \\
\hline Edi-0 & 630,728 & 35,210 & 93,151 & 356 & 99.62 \\
\hline $\mathrm{Hi}-0$ & 497,688 & 157,526 & 78,702 & 164 & 99.79 \\
\hline $\mathrm{Kn}-0$ & 637,034 & 43,211 & 98,204 & 265 & 99.73 \\
\hline Ler-0 & 647,094 & 42,652 & 98,421 & 256 & 99.74 \\
\hline Mt-0 & 588,481 & 41,237 & 84,438 & 266 & 99.69 \\
\hline No-0 & 611,346 & 45,886 & 87,517 & 233 & 99.73 \\
\hline Oy-0 & 639,949 & 42,316 & 83,055 & 287 & 99.65 \\
\hline Po-0 & 446,422 & 267,439 & 57,604 & 688 & 98.82 \\
\hline Rsch-4 & 584,081 & 44,225 & 76,089 & 222 & 99.71 \\
\hline Sf-2 & 671,638 & 72,661 & 87,741 & 234 & 99.73 \\
\hline Tsu-0 & 615,062 & 43,030 & 85,738 & 243 & 99.72 \\
\hline Wil-2 & 661,673 & 48,184 & 89,111 & 316 & 99.65 \\
\hline Ws-0 & 652,654 & 47,181 & 84,478 & 207 & 99.76 \\
\hline Wu-0 & 592,611 & 47,585 & 82,864 & 227 & 99.73 \\
\hline $\mathrm{Zu}-0$ & 631,624 & 44,391 & 92,757 & 219 & 99.76 \\
\hline Nonredundant & $\mathrm{SNPs}=3,0$ & 1,117 & SNPs wi & NA-seq su & port $=503,825$ \\
\hline
\end{tabular}




\section{Materials and Methods:}

\section{Plant material and treatments}

The seeds and leaves of Tibet- 0 were collected in the wild forest of Gongga County (N90.98, E29.3, altitude: 4200), Tibet in 2013 when it had blossomed and borne fruit. The leaves were immediately dried with silica gel and stored in sealed bags for the following molecular identification. We then grew the Tibet- 0 under a $16 \mathrm{~h}$ light $\left(22^{\circ} \mathrm{C}\right) / 8 \mathrm{~h}$ dark $\left(18^{\circ} \mathrm{C}\right)$ photoperiod regime and collected the flowers and leaves for further tests and sequencing.

We also used seeds of Col- 0 , Ler- 0 , Bur- 0 , Ws- 0 , Can- 0 and Tibet- 0 in the common garden experiments. There are 6 replicates of Can- 0 and 13 replicates of each of the other accessions. Tibet- 0 and five other $A$. thaliana ecotypes were grown at $22^{\circ} \mathrm{C}$ under a $16 \mathrm{~h}$ light $/ 8 \mathrm{~h}$ dark photoperiod regime. The phenotypes of the five ecotypes from germination to fruit were recorded and compared.

\section{Molecular Identification by gene barcoding}

In order to prove Tibet- 0 to be $A$. thaliana, we compared the nuclear internal transcribed spacer (ITS), four chloroplast genes (matK, rbcL, rpoB, rps16) and two chloroplast intergenic spacers (IGS) (trnL-trnF, trnT-trnL) between A. thaliana and A. lyrata (Online, Simon, Trajanoski et al. 2012). The total DNA was extracted by a TIANGEN Plant Genomic DNA Kit. We used the universal primers of each barcoding sequence in PCR amplification. The PCR reaction system contained 10ng DNA template, $2 \mathrm{ul}(10 \mu \mathrm{M})$ forward-reverse primers, $25 \mu \mathrm{l} 2 \mathrm{x}$ Taq PCR Master Mix (Tiangen Biotech). The PCR procedure consisted of one cycle of $94^{\circ} \mathrm{C} 5 \mathrm{~min}, 32$ cycles of $94^{\circ} \mathrm{C} 1 \mathrm{~min}, \mathrm{Ta}^{\circ} \mathrm{C}$ (Supplementary Table 1) $1 \mathrm{~min}$, and $72^{\circ} \mathrm{C} 1.5 \mathrm{~min}$, and one cycle of $72^{\circ} \mathrm{C} 7 \mathrm{~min}$ and $10^{\circ} \mathrm{C} 10 \mathrm{~min}$. The PCR products were sequenced using an ABI 3730 automated DNA sequencer (Applied Biosystems, Foster City, California, USA). We then compared all the 7 sequences of the Tibetan sample with the homologous sequence of Col-0 and Arabidopsis lyrata downloaded from NCBI by alignment using CLUSTALX (Thompson, Gibson et al. 1997). We also tested coverage and identity of those genes in the Tibetan sample, Col-0, and A. lyrata using the Blast tool of NCBI (http://blast.ncbi.nlm.nih.gov/Blast.cgi).

\section{Polyploidy determination}

To determine the polyploidy of the new Arabidopsis thaliana, the seeds of $A$. thaliana ecotypes Columbia and Tibetan were grown on filter paper with distilled water at $22^{\circ} \mathrm{C}$ with $16 \mathrm{hr}$ light $/ 8 \mathrm{hr}$ dark cycles. The seedlings $(4-5$ days old, about $1 \mathrm{~cm}$ long) were transferred on filter paper with $0.002 \mathrm{M}$ hydroxyquinoline solution for 2 hours at room temperature and for 2 hours at $4{ }^{\circ} \mathrm{C}$ in the dark. Roots of seedlings were 
submerged in hydroxyquinoline solution. The root tips were isolated and fixed in ethanol/glacial acetic acid (3:1) for one hour at room temperature and overnight at $4^{\circ} \mathrm{C}$. The root tissue was then incubated in carbolfuchsin solution for 2-3 hours at room temperature. The root tips were transferred onto a slide, and covered by a cover slip. Most of the residual staining solution was removed by once strongly pressing a flat hand on the slide (with cover slip) wrapped with filter paper. The preparations were viewed in a light microscope.

The pollen of Tibet- 0 was also observed. Seeds of wild Tibet-0 were grown under a $16 \mathrm{~h}$ light $\left(22^{\circ} \mathrm{C}\right) / 8 \mathrm{~h}$ dark $\left(18^{\circ} \mathrm{C}\right)$ photoperiod regime until blossoming occurred. The flower bud of Tibet- 0 (about $0.8-1 \mathrm{~mm}$ ) was collected at $10 \mathrm{am}$, fixed with Carony's fixative (ethanol: acetic acid $=3: 1$ ) for $24 \mathrm{~h}$ and then stored in $70 \%$ alcohol at $4{ }^{\circ} \mathrm{C}$. Following cleaning with distilled water, the pollen mother cell was then squeezed out from anther to a slide, with one drop of mixed enzyme containing $0.3 \%$ cellulase, $0.3 \%$ pectinase, and $0.5 \%$ snailase at $37^{\circ} \mathrm{C}$ for $10 \mathrm{~min}$. Using filter paper to remove the excess enzyme solution, we then added a drop of dyeing liquid (1.5ug/ml DAPI, H-1500.vector) and covered the sample with coverslips, pressing it slightly. After staining in dark for $10 \mathrm{~min}$, we observed and photographed the sample under a fluorescence microscope.

\section{Genome-wide resequencing}

The total DNA was extracted by a TIANGEN Plant Genomic DNA Kit, and genome-wide sequenced with a mean coverage of 40x the reference genomes Col-0, TAIR10 which are available at ftp://ftp.arabidopsis.org/Genes/TAIR10_genome_release/, by an Illumina HiSeq 2000 system.

\section{Sequence preparation and orthologus identification}

The gene annotation of the reference genome of $A$. thaliana was downloaded from https://www.arabidopsis.org. The CDs sequence of other $47 \mathrm{~A}$. thaliana ecotypes including Col-0, Bur-0, Ct-1, Edi-0, Hi-0, Kn-0, Ler-0, Mt-0, No-0, Po-0, Oy-0, Rsch-4, Sf-2, Tsu-0, Wil-2, Ws-0, Wu-0, Zu-0, Kas-1, Kas-2, Altai-5 and 26 relicts including Qar-8a, Etna-2, IP-Alm-0, IP-Cor-0, IP-Sne-0, IP-Con-0, Don-0, IP-Per-0, IP-Gra-0, IP-Moj-0, IP-Her-12, IP-Lso-0, IP-Cat-0, IP-Mar-1, IP-Vis-0, IP-Cem-0, IP-Vim-0, IP-Hum-2, Ped-0, IP-Nac-0, IP-Pun-0, IP-Ven-0, IP-Fun-0, Can-0, Cvi-0, IP-Iso-4 (Genomes Consortium. Electronic address and Genomes 2016) were downloaded from http://1001genomes.org/. The A. lyrata is an outcrossing perennial relative of $A$. thaliana. The sequence of A. lyrata was downloaded from Phytozome v9.1 (http://www.phytozome.net/) as outgroup. We first concatenated the CDS sequence of each gene according to its direction. We then searched the 
orthologous genes of the Tibet-0, 47 other $A$. thaliana ecotypes (26 relicts, Col-0, Bur-0, Can-0, Ct-1, Edi-0 Hi-0, Kn-0, Ler-0, Mt-0, No-0, Po-0, Oy-0, Rsch-4, Sf-2, Tsu-0, Wil-2, Ws-0, Wu-0, and $\mathrm{Zu}-0$ ) and A. lyrata by a bidirectional-best-hit method in BLAST, and obtained a total of 5741 single-copy orthologous genes. The genes of which less than half of the total CDS length were covered were then eliminated. Finally, we aligned 5611 orthologous genes in 48 A. thaliana ecotypes.

\section{Nucleotide Diversity}

The scaled mutation rate $\theta \omega=4 \mathrm{Ne} \mu$ was estimated by using the proportion of segregation sites $\theta \mathrm{s}$ (Watterson 1975) and the average pairwise nucleotide diversity $\theta \pi$ (Tajima 1989), which derived to the Tajima's D to demonstrate the distribution of the segregating sites. Putative genes were included in the analyses only if resequence data covered more than $50 \%$ of the putative coding sequences from all the accessions. As a result, a total of 5611 single copy orthologous genes of $48 \mathrm{~A}$. thaliana ecotypes were applied in the calculation. Among which, 103 genes containing no segregating sites were eliminated in the Tajima's D deduction. Analyses were conducted of the tajima_d function provided from DendroPy Python library (Sukumaran and Holder 2010), available R codes, or custom R or Python scripts.

\section{Phylogenetic analysis}

We aligned each of the 5611 orthologues genes of 48 A. thaliana ecotypes and concatenated them to long orthologous alignments to construct the maximum-likelihood (ML) tree, using RAxML v8.0 (Stamatakis 2014). The ML tree applied a partition model to take account of the different evolution rates of the first, second and third position of the codon. GTRGAMMA model was used for nucleotide substitution model. The confidence of the tree topology was evaluated by the bootstrap method with 100 replications. The time of the most recent common ancestor (tMRCA) was calculated by the Bayesian coalescent method with the BP\&P program (Rannala and Yang 2003, Yang and Rannala 2010). We then verified the accuracy of the phylogenetic tree by calculating the time of the most recent common ancestor (tMRCA) by the coalescent method (Song, Liu et al. 2012, Nakagome, Nakajima et al. 2013). Single-copy orthologous genes in 47 species and longer than $1000 \mathrm{bp}$ were extracted, and the tMRCA48 value of each gene was obtained using Bpp software (Yang 2015). We then removed each ecotype to form 48 new datasets and calculated the tMRCA47 values. For each dataset, the difference between each tMRCA47 and tMRCA48 was detected by using one tail distribution pairwise t-test.

\section{Divergence time estimation}


We used two steps to estimate the divergence time of Tibet- 0 and other ecotypes. First, we downloaded the published genome sequence of 23 species including 21 flowering, 1 fern (Selaginella moellendorffii) and 1 moss (Physcomitrella patens) from Phytozome v9.1 (http://www.phytozome.net/) (Goodstein, Shu et al. 2012) before March. 24th, 2014, and downloaded the whole genome sequence of Norway spruce (Picea abies) from Spruce Genome Project (ftp://congenie.org/congenie/) (Supplementary Table 2). After bidirectional blast between the sequence of these 24 species and the reference genome of Col-0, and removing paralogue contamination, we obtained 334 single-copy orthologous genes shared by 22 species or more. We concatenated the aligned 334 genes in each species and used '?' to fill the deletion. Based on the nucleotide and amino acid sequence super matrix, the phylogenetic ML tree was then constructed using RAxML v8.0 (Stamatakis 2014). The nucleotide ML tree applied a partition model to normalize the difference between evolution rates of the first, second and third position of the codon, and the model for nucleotide substitution rate was $\mathrm{GTR}+\mathrm{I}+\mathrm{G}$, while the amino acid ML tree was constructed using a GAMMA+LG4XF amino acid substitution rate model. Both trees were bootstrapped for 1000 times. The divergence time was estimated by using MCMCTREE package in PAML software (Yang and Rannala 2006, Rannala and Yang 2007). The model for nucleotide sequence is a GTR substitution rate model, while the model for amino acid sequence is a F84 substitution rate model. We used the relative rate test and the likelihood ratio test to detect each branch of the phylogenetic tree. In this study, 7 fossil calibrations were used to correct the divergence time of each branch (Supplementary Table 3), thus obtain the divergence time between $A$. thaliana and A. lyrata.

Secondly, we estimated the time of common ancestor between Tibet- 0 and the other 47 ecotypes using BP\&P software based on Bayesian coalescent models (Yang 2002, Rannala and Yang 2003, Burgess and Yang 2008, Yang and Rannala 2010). By using ratio of the tau of Tibet- 0 minus other thaliana accessions and the tau of the $A$. thaliana minus A. lyrata, we obtained the ratio of the divergence time between the $A$. thaliana and the A. lyrata and the divergence time between Tibet-0 and other $A$. thaliana accessions. Since we already obtained the divergence time between $A$. thaliana and A. lyrata, the divergence time between the Tibet- 0 and other accessions could be calculated by multiplying the ratio and the divergence time between $A$. thaliana and A. lyrata.

\section{Positive selection detection}

The genomic DNA reads of other 19 A. thaliana ecotypes including Col-0, Bur-0, Can-0, Ct-1, Edi-0, Hi-0, Kn-0, Ler-0, Mt-0, No-0, Po-0, Oy-0, Rsch-4, Sf-2, Tsu-0, Wil-2, Ws- $0, \quad \mathrm{Wu}-0$, and $\mathrm{Zu}-0$ were downloaded from 
http://mus.well.ox.ac.uk/19genomes/ (Gan, Stegle et al. 2011), which are also deposited in the European Nucleotide Archive (www.ebi.ac.uk/ena/) under accession number ERP000565(Gan, Stegle et al. 2011). According to the difference between Tibet-0 and other ecotypes shown in the common garden experiments, we investigated the potential adaptive evolution on the relative genes by using the CODEML package in the PAML software (Yang 2007). We applied the "branch-site" model that divided the branches of phylogenetic trees into foreground and background (Yang 1998). In order to detect if the genes in Tibet-0 have experienced positive selection, we regarded Tibet- 0 as the foreground branch, with the other 19 ecotypes the background branches. If an enzyme has experienced positive selection, we searched for its protein structure in the PDB database (http://www.rcsb.org/pdb/home/home.do), and observed whether its structure was affected by the selection by using Pymol (http://www.pymol.org).

\section{Supplementary Reference}

Burgess, R. and Z. Yang (2008). "Estimation of hominoid ancestral population sizes under bayesian coalescent models incorporating mutation rate variation and sequencing errors." Mol Biol Evol 25(9): 1979-1994.

Gan, X., O. Stegle, J. Behr, J. G. Steffen, P. Drewe, K. L. Hildebrand, R. Lyngsoe, S. J. Schultheiss, E. J. Osborne, V. T. Sreedharan, A. Kahles, R. Bohnert, G. Jean, P. Derwent, P. Kersey, E. J. Belfield, N. P. Harberd, E. Kemen, C. Toomajian, P. X. Kover, R. M. Clark, G. Ratsch and R. Mott (2011). "Multiple reference genomes and transcriptomes for Arabidopsis thaliana." Nature 477(7365): 419-423.

Genomes Consortium. Electronic address, m. n. g. o. a. a. and C. Genomes (2016). "1,135 Genomes Reveal the Global Pattern of Polymorphism in Arabidopsis thaliana." Cell 166(2): 481-491.

Goodstein, D. M., S. Shu, R. Howson, R. Neupane, R. D. Hayes, J. Fazo, T. Mitros, W. Dirks, U. Hellsten, N. Putnam and D. S. Rokhsar (2012). "Phytozome: a comparative platform for green plant genomics." Nucleic Acids Res 40(Database issue): D1178-1186.

Nakagome, S., Y. Nakajima and S. Mano (2013). "Biogeography revealed by mariner-like transposable element sequences via a Bayesian coalescent approach." J Mol Evol 77(3): 64-69.

Online, S. s. m. o. S.

Rannala, B. and Z. Yang (2003). "Bayes estimation of species divergence times and ancestral population sizes using DNA sequences from multiple loci." Genetics 164(4): 1645-1656.

Rannala, B. and Z. Yang (2007). "Inferring speciation times under an episodic molecular clock." Syst Biol 56(3): 453-466.

Simon, U. K., S. Trajanoski, T. Kroneis, P. Sedlmayr, C. Guelly and H. Guttenberger (2012). "Accession-specific haplotypes of the internal transcribed spacer region in 
Arabidopsis thaliana--a means for barcoding populations." Mol Biol Evol 29(9): 2231-2239.

Song, S., L. Liu, S. V. Edwards and S. Wu (2012). "Resolving conflict in eutherian mammal phylogeny using phylogenomics and the multispecies coalescent model." Proc Natl Acad Sci U S A 109(37): 14942-14947.

Stamatakis, A. (2014). "RAxML version 8: a tool for phylogenetic analysis and post-analysis of large phylogenies." Bioinformatics 30(9): 1312-1313.

Sukumaran, J. and M. T. Holder (2010). "DendroPy: a Python library for phylogenetic computing." Bioinformatics 26(12): 1569-1571.

Tajima, F. (1989). "Statistical method for testing the neutral mutation hypothesis by DNA polymorphism." Genetics 123(3): 585-595.

Thompson, J. D., T. J. Gibson, F. Plewniak, F. Jeanmougin and D. G. Higgins (1997). "The CLUSTAL_X windows interface: flexible strategies for multiple sequence alignment aided by quality analysis tools." Nucleic Acids Res 25(24): 4876-4882.

Watterson, G. A. (1975). "On the number of segregating sites in genetical models without recombination." Theor Popul Biol 7(2): 256-276.

Yang, Z. (1998). "On the best evolutionary rate for phylogenetic analysis." Syst Biol 47(1): 125-133.

Yang, Z. (2002). "Likelihood and Bayes estimation of ancestral population sizes in hominoids using data from multiple loci." Genetics 162(4): 1811-1823.

Yang, Z. (2007). "PAML 4: phylogenetic analysis by maximum likelihood." Mol Biol Evol 24(8): 1586-1591.

Yang, Z. (2015). "A tutorial of BPP for species tree estimation and species delimitation " Current Zoology 61: 854-865.

Yang, Z. and B. Rannala (2006). "Bayesian estimation of species divergence times under a molecular clock using multiple fossil calibrations with soft bounds." Mol Biol Evol 23(1): 212-226.

Yang, Z. and B. Rannala (2010). "Bayesian species delimitation using multilocus sequence data." Proc Natl Acad Sci U S A 107(20): 9264-9269. 